\title{
Giant cell tumour of the hand presenting as trauma to the ED
}

\author{
Muhammad N Qureshi ${ }^{1 *}$ and Dr Shahad Alsuwailem ${ }^{2}$ \\ ${ }^{1}$ Consultant Emergency Medicine \& Research Director, King Faisal Specialist Hospital and Research centre, Riyadh, KSA \\ ${ }^{2}$ Medical Intern, Alfaisal University, Riyadh, KSA
}

\section{Case}

A 30-year-old man presents to emergency department (ED) with complaints of pain \& swelling over the left index finger extending to the dorsum of the hand. He had history of trauma to the same hand few months ago, followed by onset of this swelling. It had progressively increased in size and became painful. The patient had a previous history of psychiatric disorder and epilepsy. There were no previous injuries to his hand or any condition known to affect the musculoskeletal system.

Examination revealed a hard swelling with no tenderness but restricted movements of the $2^{\text {nd }}$ metacarpophalangeal (MCP) joint and the proximal interphalangeal (PIP) joint of the left index finger. X-ray of the hand, showed a suspicious lesion of the left second metacarpal bone and the proximal phalanx without any fractures.

Patients MRI scan showed an expansile lytic lesion. Bone biopsy confirmed "Giant cell tumor of the bone" (GCT). (Figures 1 and 2)

\section{Introduction}

Giant cell tumours (GCTs) are common benign solitary tumours. They usually occur in young adults between $20-40$ years of age with a peak incidence in their 30 s with a female-to-male ratio of 1:5 [1]. Giant cell tumours of the bone typically represent $4-10 \%$ of all primary bone tumours and $15-20 \%$ of all benign bone tumours [2]. Most of GCTs present around the knee joint (50-65\%), commonly in distal femur [3]. Less common locations are in distal radius, proximal humerus, hands and feet [4] (Figures 3 and 4).

\section{Pathophysiology}

The cause of these tumours is still unclear, whether they are a true neoplasm or a reactive condition [1]. GCTs usually grow on the epiphyseal end of the long bones. They can be well-defined and eccentric in location. Most of them are focal tumours (staying to the bone of origin), a smaller proportion show aggression with extension in the surrounding soft tissues. They are also known to metastasise, mainly to the pulmonary tissue [5].

\section{Radiological features}

Plain X-ray is usually the first modality of imaging, which shows their anatomical location. Cortical thinning, radiolucency of lesion and bone destruction are usually the characteristic features [5].

Computerised Tomography (CT) shows more detailed features of these tumours including the full extent of the lesion, cortical thinning and penetration.

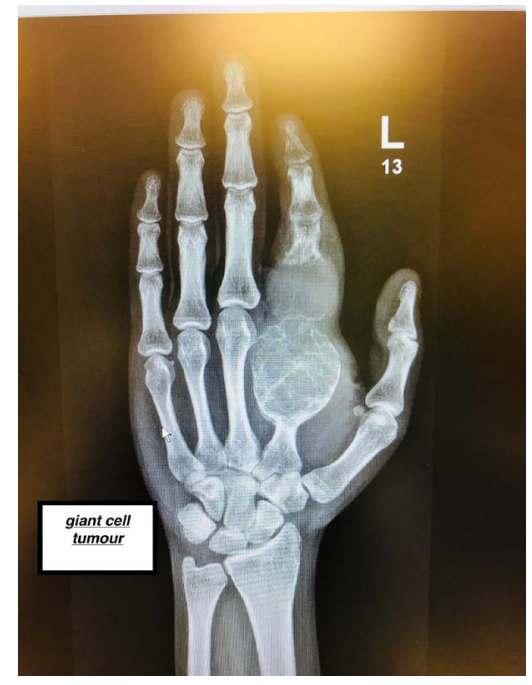

Figure 1. Xray GCT1

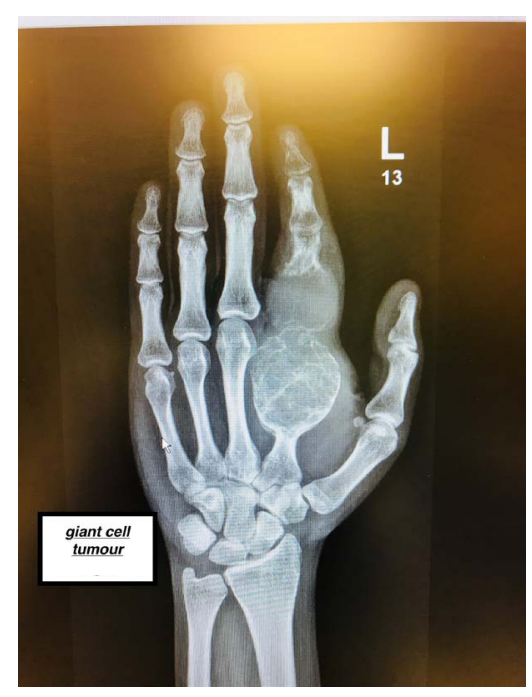

Figure 2. Xray GCT2

${ }^{\star}$ Correspondence to: Muhammad Qureshi, Consultant Emergency Medicine \& Research Director, King Faisal Specialist hospital \& Research center, Riyadh, KSA, E-mail: qmuhammad@kfshrc.edu.sa

Received: November 30, 2020; Accepted: December 21, 2020; Published: December 24, 2020 


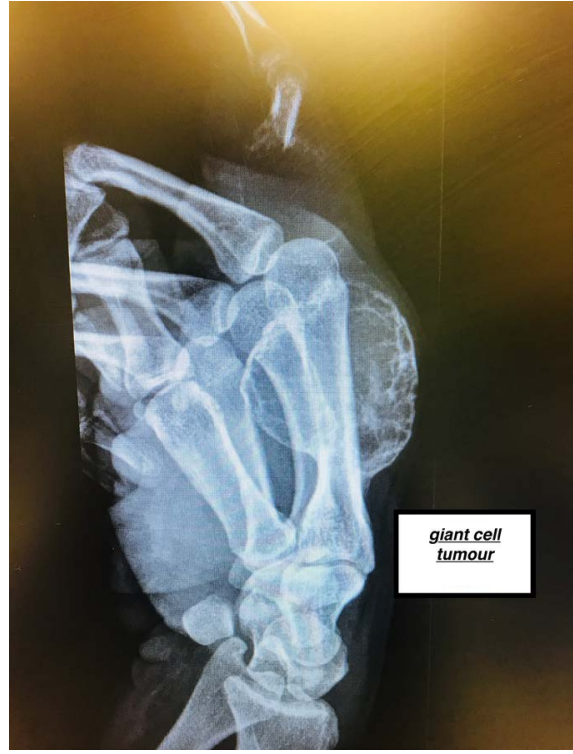

Figure 3. Xray GCT3

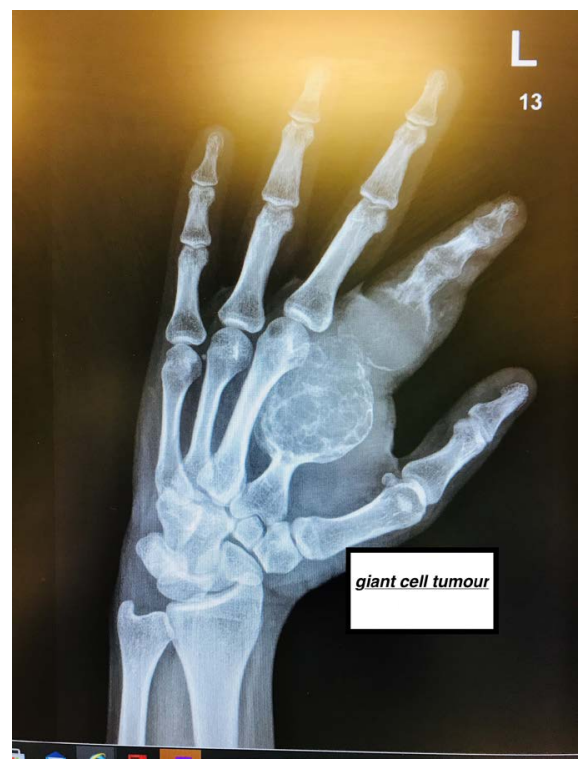

Figure 4. Xray GCT4

Magnetic resonant imaging (MRI) shows not only the extent of the primary bony lesion but also its effect on the surrounding soft tissues [6,7] (MRI $1 \&$ MRI 2).

\section{Presenting features}

The typical clinical presentation of these tumours is pain, swelling and limitation of joint movement. Reactive joint effusions can also be a feature [6].

\section{Diagnostic work up}

Once the clinical suspicion has been raised, imaging modalities are usually deployed in a step wise manner, starting with a plain Xrays followed by CT and MRI [1-5]. These imaging modalities help determine the characteristics of GCT, stage of the tumour and screen for metastasis [4-6]. To reach a definitive diagnosis, biopsy is essential [2-4]. (MRI 3 \& MRI 4)

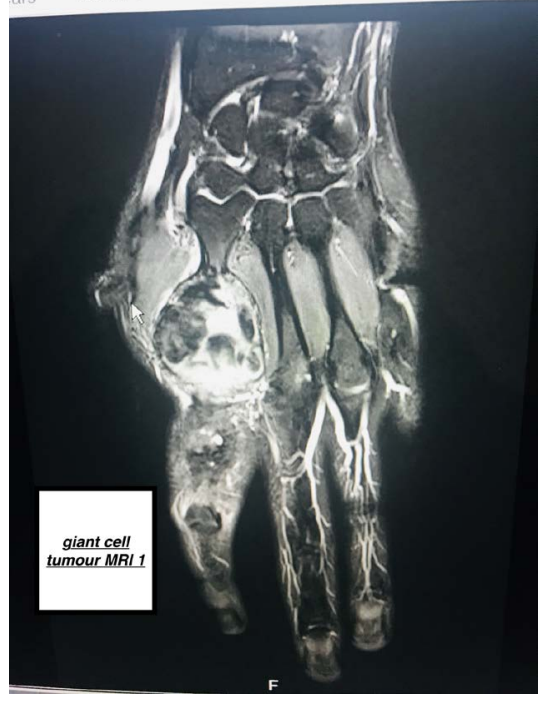

MRI 1. MRI GCT1

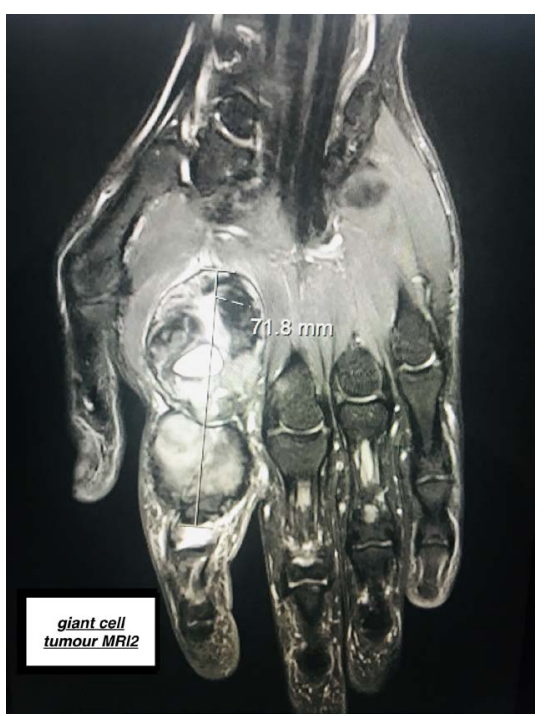

MRI 2. MRI GCT2

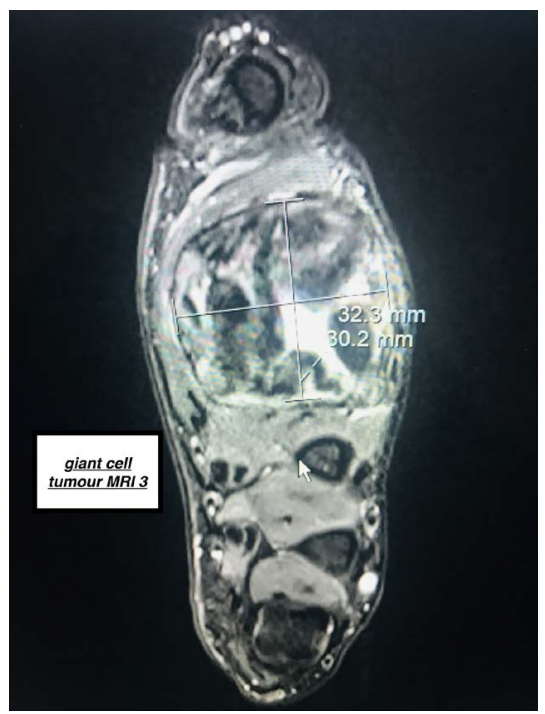

MRI 3. MRI GCT3 


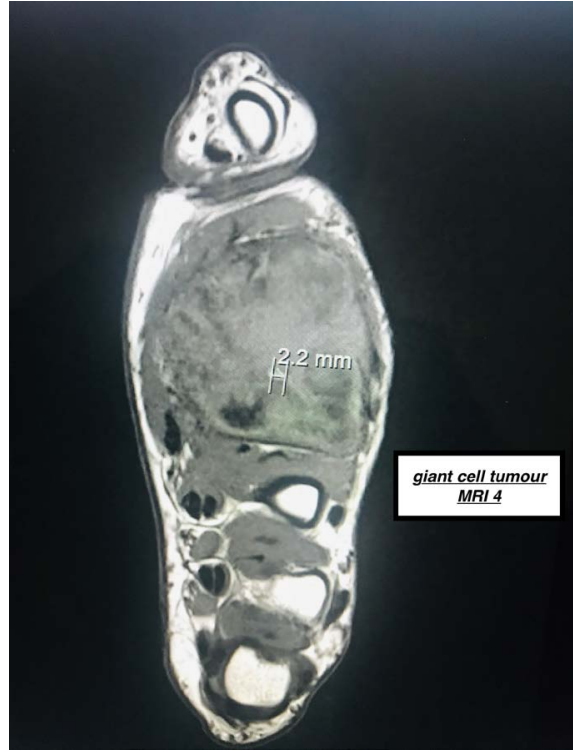

MRI 4. MRI GCT4

\section{Treatment modalities}

Surgical resection is the main stay treatment for GCTs [1-6]. It can take the form of curative surgery with wide excision or intra-lesional curettage. The former is more amenable to prevent recurrence whilst the later leads to less functional impairment. Intra-lesional curettage (with or without bone graft) is usually preferred due to the benign nature of the tumour.

In addition, certain adjuvant therapies can be used in treating these tumours, such as liquid nitrogen, acrylic cement, phenol and hydrogen peroxide. These work very well in conjunction with aggressive intralesional curettage [1-5].

Radiotherapy can be used for GCT involving wide bony surfaces like pelvis and sacrum, especially when complete excision is not possible [4].

Denosumab is a human monoclonal antibody for treatment of giant cell tumours. It binds to RANKL (receptor activator of NFkB ligand), preventing RANKL from activating RANK, its receptor on the osteoclast surface. With reduced RANK-RANKL binding, osteoclast formation and survival are inhibited, bone resorption decreases and bone mass increases [1-3].

Bisphosphonates bind bone mineral, where they are absorbed by osteoclasts, inducing osteoclast apoptosis and suppressing resorption [4-6].

\section{Prognosis}

These tumours have a recurrence rate of $20-50 \%$ but are less likely to be metastatic [1].

\section{Discussion}

Trauma is a rare presentation of a tumour in ED. In our report, GCT of the of the small bones of the left hand, presented to ED triage, as a case of minor trauma. It is unclear whether the trauma was a coincidence or it genuinely contributed to the initiation or activation of the tumour. Pathological fractures can be a secondary consequence of tumours [7].

Our reported GCT can be classed as Grade III (aggressive tumour with extra-osseous extension), as it had been growing over the past few months till it became cosmetically visible. The patients' psychiatric illness might have contributed towards delay in presentation [1,2].
The site of the reported tumour is also relatively uncommon. Most of them present around the knee joint [8].

The expansile nature of the tumours can cause significant functional impairment Our patients' tumour luckily did not significantly impact on the functionality of the index finger, as the distal inter phalangeal joint (DIP) could still be actively moved. This could be due to sparing of the soft tissues, against the conventional nature of GCTs [9].

Majority of the tumours present in a primary care setting, where an onward referral is made to an outpatient clinic for further investigation. In $\mathrm{ED}$, fracture is usually an expectation in a limb trauma presentation. A high index of clinical suspicion with a targeted use of investigations is key for diagnosing uncommon presentations, in a time limited ED environment [11]

Unexpected X-ray finding in ED should lead to a careful and focused communication with the patient. The communicator should refrain from giving a presumptive diagnosis and emphasise on the need of further investigation in an outpatient setting [11].

There should be an expedited referral pathway from ED to an outpatient specialty clinic for triggering conclusive diagnostic tests. Our patient was fast tracked to orthopaedic oncology clinic where the definitive diagnosis was reached within 2 weeks from the time of presentation.

The series of investigations carried out included MRI of the hand, positron emission tomography (PET) scan followed by MRI guided bone biopsy to establish the histological diagnosis. A complete screening for metastasis was also carried out, which was negative [10].

Patient received a course of denosumab followed by successful curative surgical resection. There was no signs of tumour recurrence at one year follow.

\section{References}

1. Hosseinzadeh, Shayan, Orlando De Jesus (2020) Giant Cell Tumor. StatPearls.

2. Sota O, Okuda S, Sugiura H, Matsumoto S, Sasaki A, et al. (2018) Giant cell tumors of the bone: changes in image features after denosumab administration. Magnetic Resonance in Medical Sciences 17(4): 325.

3. Andreas FM, Igoumenou VG, Megaloikonomos PD, Panagopoulos GN, Papagelopoulos PJ, Soucacos PN (2017) Giant cell tumor of bone revisited. Sicot J 3: 54. [Crossref]

4. Arun SS, Chawla NS, Chawla SP (2015) Giant-cell tumor of bone: treatment options and role of denosumab. Biologics: targets \& therapy 9: 69-74. [Crossref]

5. Corey JC, Forrester DM, Gottsegen CJ, Patel DB, White EA, et al. (2013) Giant cell tumor of bone: review, mimics, and new developments in treatment. Radiographics 33 197-211. [Crossref]

6. Anshul S, Agrawal P, Agarwala S, Agarwal M (2016) Giant cell tumor of bone-an overview. Archives of Bone and Joint Surgery 4: 2-9. [Crossref]

7. Seethalakshmi V, Jambhekar NA (2010) Metastatic giant cell tumor of bone: are there associated factors and best treatment modalities? Clinical Orthopaedics and Related Research ${ }^{\circledR}$ 468: 827-33.

8. Obada H, Ali M, Mustafa M, Ali A, Umer M (2019) Treatment and recurrence of giant cell tumors of bone-A retrospective cohort from a developing country. Annals of Medicine and Surgery 48: 29-34. [Crossref]

9. Kenta M, Suehara Y, Kohsaka S, Akaike K, Tanabe Y, et al. (2016) Protein expression profiling of giant cell tumors of bone treated with denosumab. PloS one 11: $\mathrm{e} 0148401$. [Crossref]

10. Enrico WC, Jayasuriya RA, Hardy J, Bannan A, Thorpe MEC (2002) Communication loads on clinical staff in the emergency department. Medical Journal of Australia 176: 415-418. [Crossref]

11. Rollin JF, Bisantz AM, Sunm M (2007) Emergency department communication links and patterns. Annals of Emergency Medicine 50: 396-406.

Copyright: (C2020 Qureshi MN. This is an open-access article distributed under the terms of the Creative Commons Attribution License, which permits unrestricted use, distribution, and reproduction in any medium, provided the original author and source are credited. 